\title{
Shape evolution of antimony oxychloride from sheaf-like to quasi-wafer structures
}

\author{
ZHOU Jing ${ }^{1,2}$, ZHAO HeWei ${ }^{1}$, LI LiDong ${ }^{1} \&$ GUO Lin $^{1 *}$ \\ ${ }^{1}$ School of Chemistry and Environment, Beihang University, Beijing 100191, China; \\ ${ }^{2}$ School of Chemistry Engineering, Northeast Dianli University, Jilin 132012, China
}

Received May 17, 2011; accepted May 24, 2011

\begin{abstract}
Antimony oxychloride $\mathrm{Sb}_{8} \mathrm{O}_{11} \mathrm{Cl}_{2}\left(\mathrm{H}_{2} \mathrm{O}\right)_{6}$ products with various morphologies including sheaf-like, rhombic-plate, oval leaf-like and quasi-wafer have been successfully synthesized via a mild and facile solution route at room temperature. The morphologies and structures of the as-prepared samples were characterized by X-ray powder diffraction (XRD), scanning electron microscopy (SEM) and transmission electron microscopy (TEM). A possible formation mechanism of these structures is proposed according to the experimental results and analysis.
\end{abstract}

antimony oxychloride, sheaf-like, quasi-wafer, crystal splitting, shape evolution

Citation: Zhou J, Zhao H W, Li L D, et al. Shape evolution of antimony oxychloride from sheaf-like to quasi-wafer structures. Chinese Sci Bull, 2011, 56: 3817-3822, doi: 10.1007/s11434-011-4735-1

Over the past decade, an important target of material science is the design of inorganic materials with precisely tailored shapes and sizes that have novel properties (optical, electrical, magnetic and catalytic features) which are strongly dependent not only on their composition, but also on the structure, phase, shape, size and size distribution [1-8]. As a result, the synthesis of inorganic nanomaterials with well-controlled shapes and sizes has recently attracted considerable interest. Moreover, it is important to match the desired shape, size and phase with the corresponding potential applications. Consequently, by understanding or controlling the growth mechanism, an important link between the required product morphologies and the experimental parameters is made.

Among the series of $\mathrm{Sb}$ flame retardants, antimony oxychlorides ( $\mathrm{SbOCl}, \mathrm{Sb}_{4} \mathrm{O}_{5} \mathrm{Cl}_{2}, \mathrm{Sb}_{8} \mathrm{O}_{11} \mathrm{Cl}_{2}$ ) exhibit superior flame retardant properties that can be used as a flame retardant alone and also provide good synergistic effects when used with a halogenated organic compound [9]. However, there is limited information available on the preparation of the various antimony oxychloride nanostructures using a mild

*Corresponding author (email: guolin@buaa.edu.cn) and facile solution route. Currently, $\mathrm{Sb}_{4} \mathrm{O}_{5} \mathrm{Cl}_{2}$ nanobelts [10], $\mathrm{Sb}_{8} \mathrm{O}_{11} \mathrm{Cl}_{2}$ architectures such as nanobelts [10], nanowires, nanowire-flower, bundles of nanowires, nanobelts, bundles of flakes and hollow prisms [11], and needle-like $\mathrm{Sb}_{8} \mathrm{O}_{11} \mathrm{Cl}_{2^{-}}$ $\left(\mathrm{H}_{2} \mathrm{O}\right)_{6}$ microcrystallines [12], have been obtained by solution and hydrothermal methods. However, challenging synthesis problems remain, including how to solve the irregular shapes or impure-phases present in the products.

Herein, we report a mild, facile solution route of the synthesis of $\mathrm{Sb}_{8} \mathrm{O}_{11} \mathrm{Cl}_{2}\left(\mathrm{H}_{2} \mathrm{O}\right)_{6}$ with different morphologies in the presence of poly (vinyl pyrrolidone) (PVP) at room temperature. In our system, the shape evolution from sheaflike to rhombic-plate, oval leaf-like and quasi-wafer structures has been investigated using different concentrations of $\mathrm{NaOH}$, which may influence the degree of crystal splitting during the reaction. Furthermore, the mechanism of the evolution of the morphologies is postulated.

\section{Experimental section}

\subsection{Synthesis}

All of the chemical reagents used were analytical grade and 
used without further purification. In a typical procedure, $0.555 \mathrm{~g}$ polyvinyl pyrrolidone (PVP, MW 58000) and 30 $\mathrm{mL}$ of a $0.033 \mathrm{~mol} / \mathrm{L} \mathrm{SbCl}_{3}$ ethylene alcohol (EG) solution were initially added to a round-bottomed glass flask. After constant stirring, a colorless solution was acquired. Subsequently, an appropriate amount of $\mathrm{NaOH}$ at different concentrations $(0.25,0.35,0.40,0.45,0.48$ and $0.50 \mathrm{~mol} / \mathrm{L})$ was added in a dropwise manner to the above solution and the stirring was continued for $5 \mathrm{~h}$. The solution was then left standing for several hours. A white precipitate was obtained, washed several times with ethanol and finally dried at $50^{\circ} \mathrm{C}$ overnight in an oven.

\subsection{Characterization}

The as-prepared products were characterized by X-ray powder diffraction (XRD), field emission scanning electron microscopy (FESEM) and transmission electron microscopy (TEM). The XRD analysis was performed on a Rigaku Dmac $2200 \mathrm{X}$-ray diffractometer (Japan) with $\mathrm{Cu} \mathrm{K} \alpha$ radiation $(\lambda=1.5416 \AA)$ at a scanning speed of $0.02 \%$ s over the $2 \theta$ range of $10^{\circ}-60^{\circ}$. The morphologies of the samples were directly observed with SEM using a Hitachi S-4800 (Japan) at an accelerating voltage of $5 \mathrm{kV}$. TEM observations were carried out using a JEOL JEM-2100F microscope operating at $200 \mathrm{kV}$. Specific surface areas were measured at $77 \mathrm{~K}$ by a Brunauer-Emmett-Teller (BET) nitrogen adsorptiondesorption instrument (NOVA 2200e, Quanthachrome, USA.), and pore size distributions were calculated from the adsorption branch of the nitrogen adsorption-desorption isotherm using the Barrett-Joyner-Halenda (BJH) formula.

\section{Results and discussion}

\subsection{Characterization of the as-prepared samples}

X-ray powder diffraction (XRD) was used to characterize the chemical composition and phase purity of the as-prepared products. As shown in Figure 1, the diffraction peaks in all samples matched with the structure of the orthorhombic $\mathrm{Sb}_{8} \mathrm{O}_{11} \mathrm{Cl}_{2}\left(\mathrm{H}_{2} \mathrm{O}\right)_{6}$ species with lattice constants $a=$ $0.9618 \mathrm{~nm}, b=1.314 \mathrm{~nm}$ and $c=0.4078 \mathrm{~nm}$ (JCPDS No. 77-1584). The intensity ratio of the reflection peaks represents a well established approach used to discuss the crystal orientation against the standard powder XRD pattern $[13,14]$. Based on our detailed analysis, we observed that the intensity ratio between the (020) and (031) diffraction peaks gradually changed with different shapes obtained for samples in different concentrations of $\mathrm{NaOH}$. In Figure 1(a), this intensity ratio is lower than the ratio of similar peaks in the standard powder pattern (0.57 versus 0.75$)$. By careful analysis of Figure 1(b)-(e), the intensity ratios between the (020) and (031) diffraction peaks increased as the concentration of $\mathrm{NaOH}$ increased (numerical values corresponding to $0.62,0.92,1.01$ and 3.57 , respectively). Furthermore, the intensity ratios between the (040) and (031) diffraction peaks, and the (060) and (031) diffraction peaks distinctly increased in Figure 1(e). These results indicate the anisotropic growth of these planes during the growing process. The results are further confirmed by the following discussion.

The representative SEM images of the products prepared with different concentrations of $\mathrm{NaOH}$ are shown in Figure 2. Sheaf-like microcrystals of antimony oxychlorides (Figure 2(a) and (b)) with different outspread extensions at the top were formed when the concentration of $\mathrm{NaOH}$ was either 0.25 or $0.35 \mathrm{~mol} / \mathrm{L}$. Despite bearing the so-called "sheaf-like" structure with the top and bottom fanning out and the middle remaining tightly bound, the extent of the outspread in product (a) is clearly larger than observed in product (b), and the length of the structures are also longer in (a) than observed in (b).

When the $\mathrm{NaOH}$ concentration was increased to 0.40 $\mathrm{mol} / \mathrm{L}$, the morphology of the products evolved to a middle structure between a sheaf-like and a rhombic-plate shape, as shown in Figure 2(c). The main structure is the rhombic form and the two ends appear to have fork-like features.

Increasing the $\mathrm{NaOH}$ concentration to $0.45 \mathrm{~mol} / \mathrm{L}$ leads to the formation of primarily rhombic-plate structures with very few plates containing a fork-like feature at an end (Figure 4(d)). The oval leaf-like structure of $\mathrm{Sb}_{8} \mathrm{O}_{11} \mathrm{Cl}_{2}$ $\left(\mathrm{H}_{2} \mathrm{O}\right)_{6}$ is observed in Figure 2(e) when the $\mathrm{NaOH}$ concentration was increased to $0.48 \mathrm{~mol} / \mathrm{L}$. The as-prepared $\mathrm{Sb}_{8} \mathrm{O}_{11} \mathrm{Cl}_{2}\left(\mathrm{H}_{2} \mathrm{O}\right)_{6}$ crystals possess primarily an oval morphology with $\sim 3 \mu \mathrm{m}$ lengths, $\sim 1.8 \mu \mathrm{m}$ widths and $\sim 0.4 \mu \mathrm{m}$ thicknesses. The shape finally forms a quasi-wafer structure from the oval leaf structure as the $\mathrm{NaOH}$ concentration reached $0.50 \mathrm{~mol} / \mathrm{L}$ (Figure 2(f)). If the concentration of $\mathrm{NaOH}$ exceeds $0.5 \mathrm{~mol} / \mathrm{L}$, the product is $\mathrm{Sb}_{2} \mathrm{O}_{3}$ instead of $\mathrm{Sb}_{8} \mathrm{O}_{11} \mathrm{Cl}_{2}\left(\mathrm{H}_{2} \mathrm{O}\right)_{6}$, which has been discussed previously [15]. From the fully developed sheaf-like structure to a sheaflike shape with decreasing extensions at the edges, i.e.,

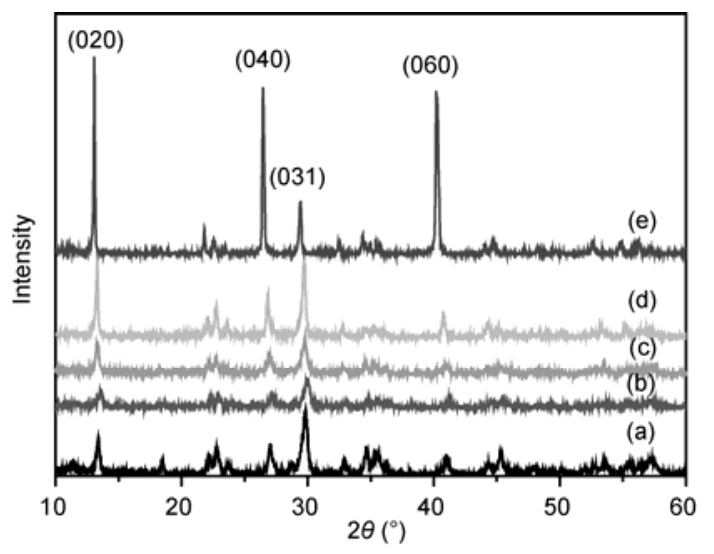

Figure 1 XRD patterns obtained from the as-synthesized samples with the various concentrations of $\mathrm{NaOH}$ : (a) 0.25 , (b) 0.35 , (c) 0.40 , (d) 0.45 and (e) $0.50 \mathrm{~mol} / \mathrm{L}$. 

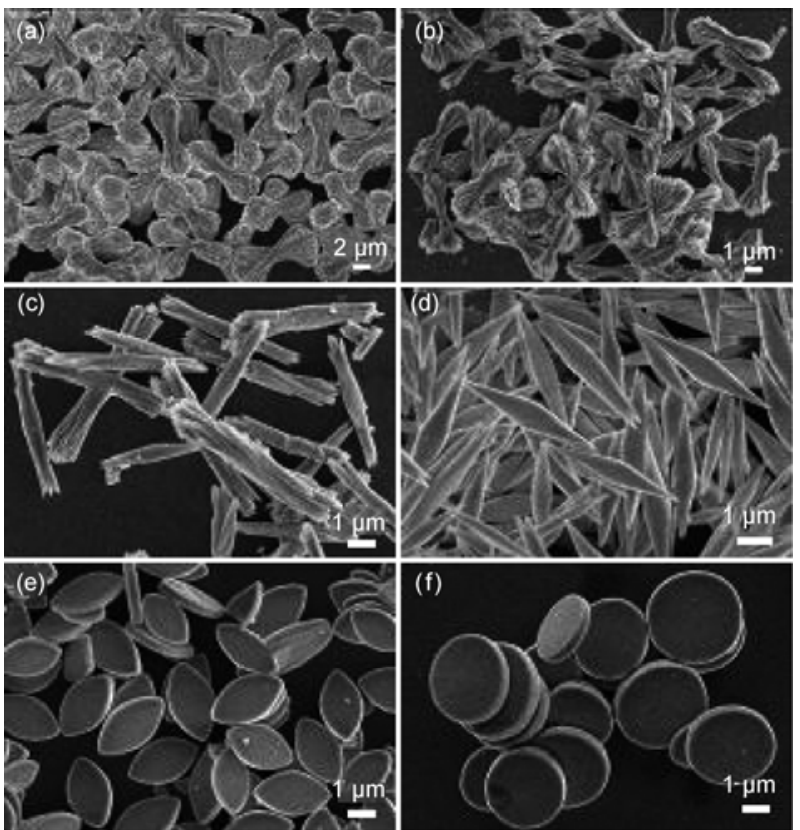

Figure 2 SEM images obtained from $\mathrm{Sb}_{8} \mathrm{O}_{11} \mathrm{Cl}_{2}\left(\mathrm{H}_{2} \mathrm{O}\right)_{6}$ samples with various concentrations of $\mathrm{NaOH}$ : (a) 0.25 , (b) 0.35 , (c) 0.40 , (d) 0.45 , (e) 0.48 and (f) $0.50 \mathrm{~mol} / \mathrm{L}$.

quasi-wafer shape, the evolution of the whole process is clearly presented.

The morphologies of the as-synthesized products using different concentrations $\mathrm{NaOH}$ were further investigated by TEM analysis, as shown in Figure 3. All images in Figure 3 clearly show the evolution of the morphologies from sheaf-like to rhombic-plate, oval leaf-like and quasi-wafer structures. The selected area electron diffraction (SAED) patterns taken from the tips of each single as-prepared product show different information (as shown in the insets of Figure 3(a)-(f)). In the insets of Figure 3(a) and (b), the SAED patterns show discrete bright elongated dots that indicate the sheaf-like microcrystals were assembled by nanorods in a highly oriented way. From Figure 3(c) to (f), the SAED patterns all show discrete spots, which clearly indicate a single crystal of the as-obtained products. The patterns could be indexed to the [020] zone axis of the monoclinic phase $\mathrm{Sb}_{8} \mathrm{O}_{11} \mathrm{Cl}_{2}\left(\mathrm{H}_{2} \mathrm{O}\right)_{6}$, and these pattern spots were consistent with the XRD results which demonstrated that the shape evolution from the sheaf-like to quasi-wafer of the as-gained products has an oriented formation.

The nitrogen adsorption-desorption isotherm curves of the $\mathrm{Sb}_{8} \mathrm{O}_{11} \mathrm{Cl}_{2}\left(\mathrm{H}_{2} \mathrm{O}\right)_{6}$ (Figure 4(a) to (c)) exhibit the type IV isotherms that are assigned to the mesostructure. The BET surface areas of the $\mathrm{Sb}_{8} \mathrm{O}_{11} \mathrm{Cl}_{2}\left(\mathrm{H}_{2} \mathrm{O}\right)_{6}$ samples with different concentrations of $\mathrm{NaOH}$ are $(9.49 \pm 0.5),(8.98 \pm 0.5)$ and $(8.47 \pm 0.5) \mathrm{m}^{2} / \mathrm{g}$, respectively. The decreasing BET surface areas with increasing concentrations of $\mathrm{NaOH}$ are consistent with the morphology observations obtained from the SEM results. To further analyze the pore structures of the samples with different concentrations of $\mathrm{NaOH}$, pore size distribution
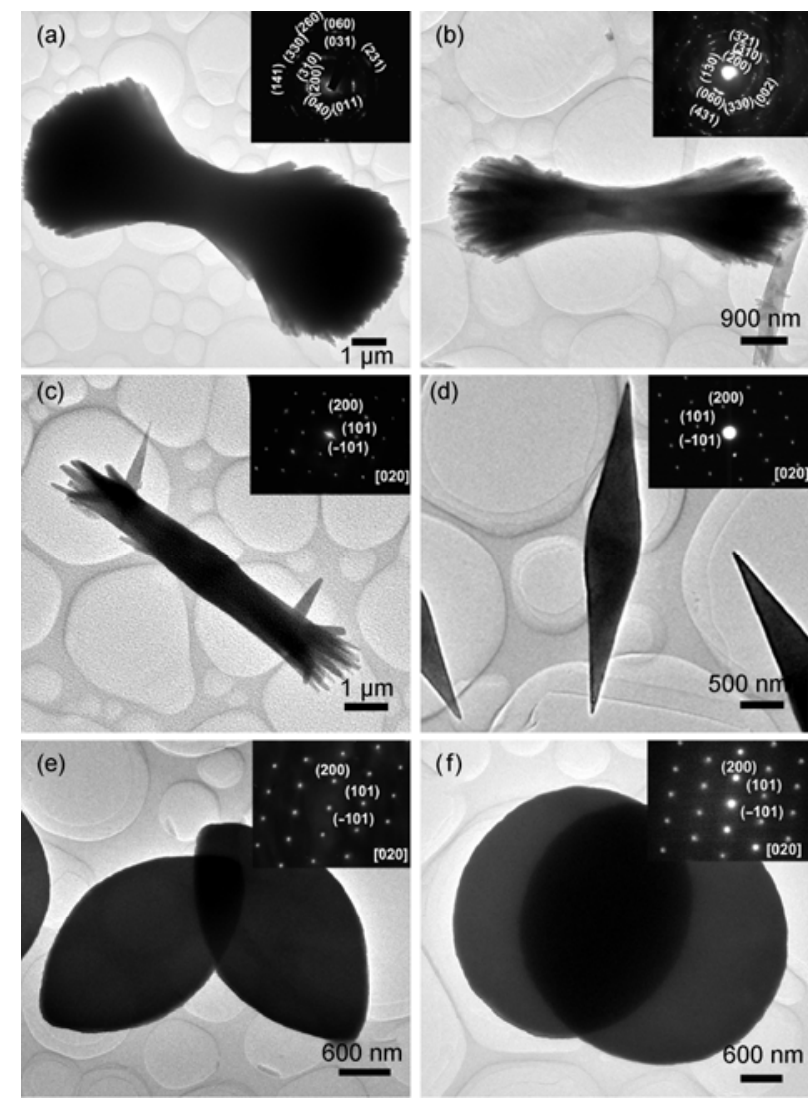

Figure 3 Representative TEM images obtained from $\mathrm{Sb}_{8} \mathrm{O}_{11} \mathrm{Cl}_{2}\left(\mathrm{H}_{2} \mathrm{O}\right)_{6}$ samples with different concentrations of $\mathrm{NaOH}$ : (a) 0.25 , (b) 0.35 , (c) 0.40 , (d) 0.45 , (e) 0.48 and (f) $0.50 \mathrm{~mol} / \mathrm{L}$. The insets in every figure show the corresponding SAED patterns.

curves using the BJH (Barrett-Joyner-Halenda) method were investigated. As presented in the insets of Figure $4(\mathrm{a})-(\mathrm{c})$, the pore sizes of $\mathrm{Sb}_{8} \mathrm{O}_{11} \mathrm{Cl}_{2}\left(\mathrm{H}_{2} \mathrm{O}\right)_{6}$ were centered at $3.83,3.06$ and $2.75 \mathrm{~nm}$ as the concentration of $\mathrm{NaOH}$ increased from 0.25 to $0.45 \mathrm{~mol} / \mathrm{L}$, indicating that the samples have mesopores.

\subsection{Growth mechanism}

As previously reported for other sheaf-like crystals, including $\mathrm{Bi}_{2} \mathrm{~S}_{3}$ [16-18], $\beta$ - $\mathrm{FeO}(\mathrm{OH})$ [19], $\mathrm{BaWO}_{4}$ [20,21], lanthanum orthovanadates [22], $\mathrm{Sb}_{2} \mathrm{~S}_{3}$ and $\mathrm{Sb}_{2} \mathrm{Se}_{3}$ [23,24], $\mathrm{CuO}$ $[25,26], \mathrm{CeO}_{2}$ [27] and Tb-doped $\mathrm{CePO}_{4}$ [28], it has been widely accepted that the formation mechanism of sheaf-like morphologies is because of crystal splitting. Crystal splitting possibly occurs because of solution oversaturation conditions are used, or the presence of additives which may influence the crystal growth process leading to crystal splitting. In our synthesis, the effect of PVP is significant in constructing the sheaf-like shape. In the absence of PVP, only irregular nanorods with some fork-like features were obtained (Figure 4(a)). As a very potent crystal face inhibitor and surface stabilizer, it is postulated that the presence of PVP may kinetically control the growth rate of different 

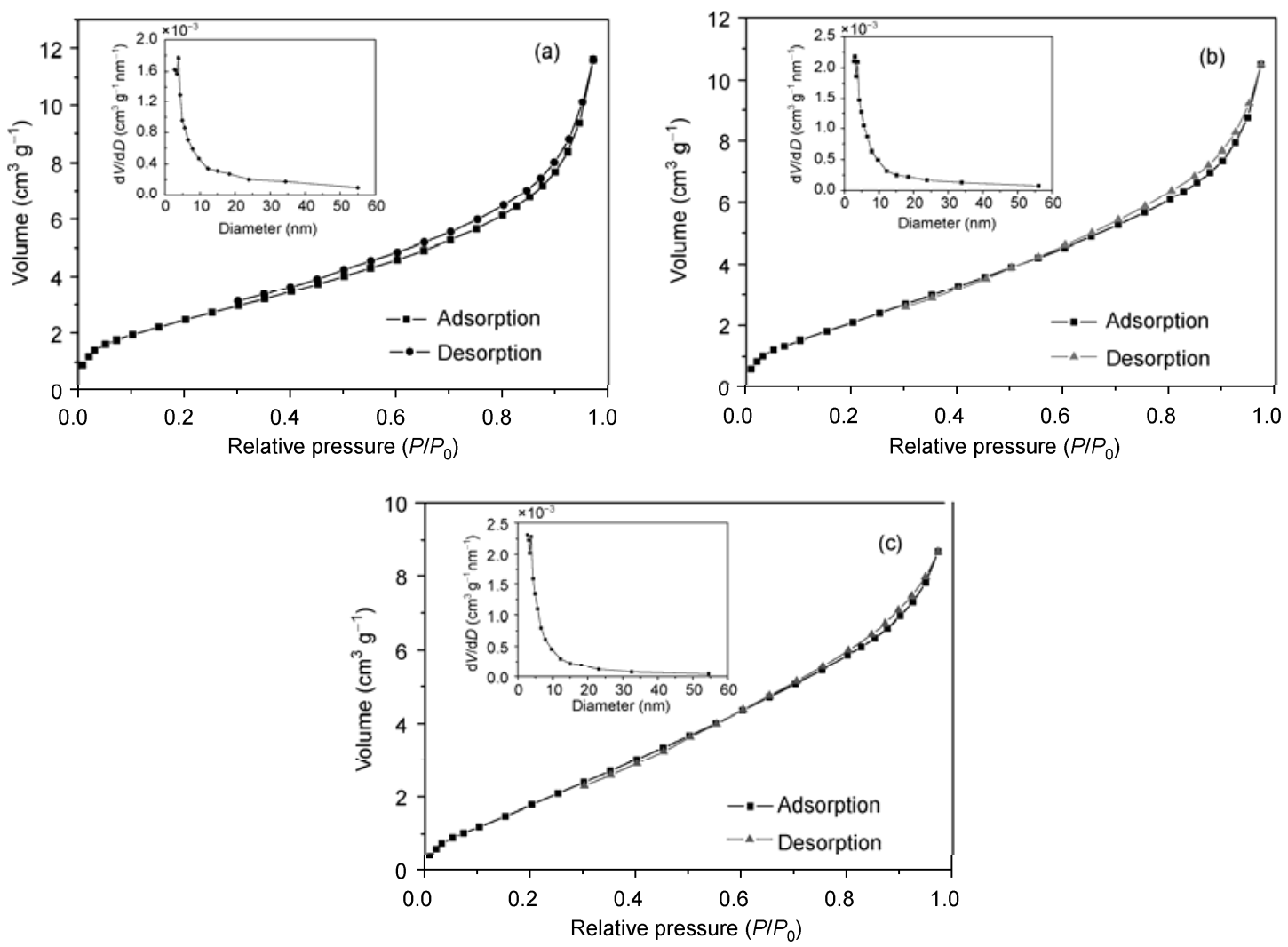

Figure 4 Nitrogen adsorption-desorption isotherm and pore size distribution of sheaf-like $\mathrm{Sb}_{8} \mathrm{O}_{11} \mathrm{Cl}_{2}\left(\mathrm{H}_{2} \mathrm{O}\right)_{6}$ microcrystals with different concentrations of $\mathrm{NaOH}$ : (a) 0.25 , (b) 0.40 , (c) $0.45 \mathrm{~mol} / \mathrm{L}$.

crystallographic faces of the crystal by selectively absorbing onto specific faces. However, we have no experimental data to clarify the certain face because of the thickness of the core part. The selective adsorption of PVP on specific faces leads to the preferential and fast growth of a particular face, and this process consequently enhances crystal splitting.

The effect of PVP in the absence of $\mathrm{NaOH}$ was examined. Figure 5 shows $\mathrm{SEM}$ images obtained from $\mathrm{Sb}_{8} \mathrm{O}_{11} \mathrm{Cl}_{2^{-}}$ $\left(\mathrm{H}_{2} \mathrm{O}\right)_{6}$ samples with different concentrations of $\mathrm{NaOH}$ in the absence of PVP. Clearly, in comparison to the previous results, when the concentration of $\mathrm{NaOH}$ was lower than $0.45 \mathrm{~mol} / \mathrm{L}$ the shapes of the as-obtained products were different from the results obtained when PVP was present. However, increasing the $\mathrm{NaOH}$ concentration to $0.45,0.48$, or $0.50 \mathrm{~mol} / \mathrm{L}$ gave similar rhombic-plate, quasi-oval or quasi-wafer morphologies, respectively.

These observations are explained by the crystal-splitting theory in conjunction with the nucleation and growth theory of nanocrystals. It is well known that crystal splitting is possible if oversaturation exceeds a certain "critical" level and the sheaf structure will form under fast crystal growth conditions. However, when the $\mathrm{NaOH}$ concentration increases, more nuclei will form in a shorter period. Consequently, a large number of nuclei will grow slowly, which prohibits the splitting growth process [18,29]. Similarly, in our experiments, the degree of crystal splitting decreases

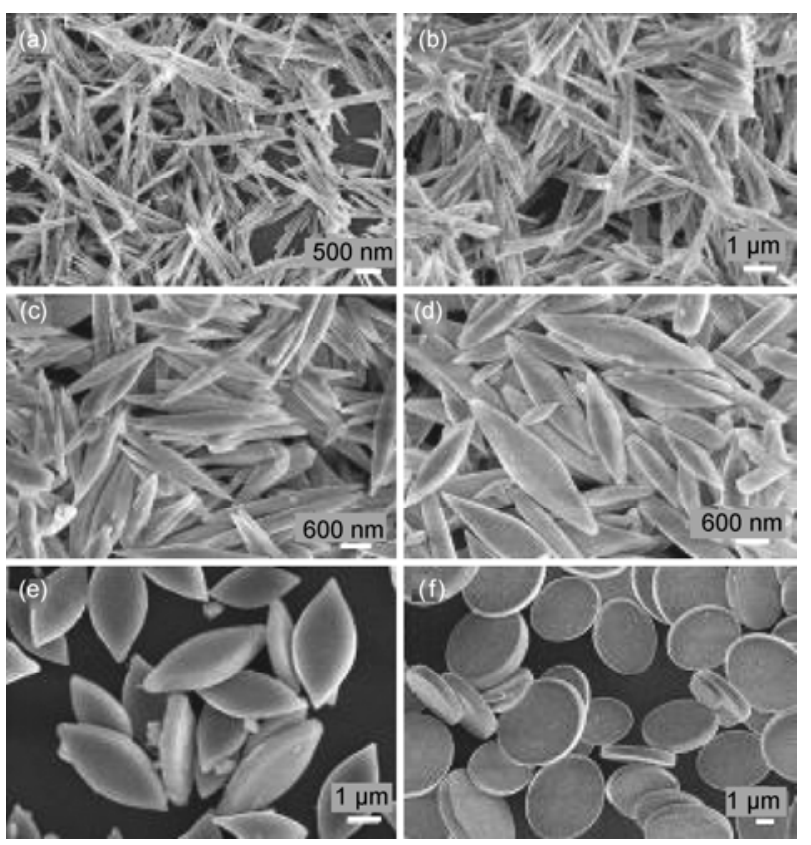

Figure 5 SEM images obtained from $\mathrm{Sb}_{8} \mathrm{O}_{11} \mathrm{Cl}_{2}\left(\mathrm{H}_{2} \mathrm{O}\right)_{6}$ samples with various concentrations of $\mathrm{NaOH}$ in the absence of PVP: (a) 0.25 , (b) 0.35 , (c) 0.40 , (d) 0.45 , (e) 0.48 and (f) $0.50 \mathrm{~mol} / \mathrm{L}$.

with increasing concentration of $\mathrm{NaOH}$ in the presence of PVP, i.e., from the sheaf structure with large numbers of 
bundles $(0.25 \mathrm{~mol} / \mathrm{L} \mathrm{NaOH})$, to bundle numbers decreasing $(0.35 \mathrm{~mol} / \mathrm{L} \mathrm{NaOH})$, to small bundles with a small degree of splitting $(0.40 \mathrm{~mol} / \mathrm{L} \mathrm{NaOH})$, to individual rhombic-plate structures with negligible splitting $(0.45 \mathrm{~mol} / \mathrm{L} \mathrm{NaOH})$, to oval leaf-like, quasi-wafer structures with no splitting $(0.48$ and $0.50 \mathrm{~mol} / \mathrm{L} \mathrm{NaOH}$, respectively). Therefore, the evolution of the morphologies is dependent on the concentration of the reactant in the solution system in the presence of PVP. It is very clear that the shapes of the as-obtained products were different when the concentration of $\mathrm{NaOH}$ was lower than $0.45 \mathrm{~mol} / \mathrm{L}$ in the absent of PVP. In contrast, when the concentration of $\mathrm{NaOH}$ was $>0.45 \mathrm{~mol} / \mathrm{L}$, the as-obtained results are similar to those obtained when PVP is present. Therefore, we can assume that when the concentration of $\mathrm{NaOH}$ is lower than $0.45 \mathrm{~mol} / \mathrm{L}$ the function of PVP is important for forming the sheaf-like structures with different degrees of crystal splitting. By increasing the concentration of $\mathrm{NaOH}$ to $\geqslant 0.45 \mathrm{~mol} / \mathrm{L}$, the effect of the $\mathrm{NaOH}$ concentration is identical to that observed in the presence of PVP. More nuclei will form in a shorter period and grow slowly, which results in structures with no splitting. This observation is also supported by the results examining a change in the molar amount of $\mathrm{Sb}^{3+}$ in system, as observed in Figure 6. From Figure 6(a) to (f), with decreasing concentrations of $\mathrm{Sb}^{3+}$, the shape evolution from sheaf-like to rhombic-plate, oval leaf-like and quasi-wafer structure is clearly observed. This observation is in agreement with the conditions in which the $\mathrm{Sb}^{3+}$ molar amount is held constant, but the concentration of $\mathrm{NaOH}$ is modulated.

Based on the above investigation, it can be deduced that the presence of PVP and the ratio of reactant play important
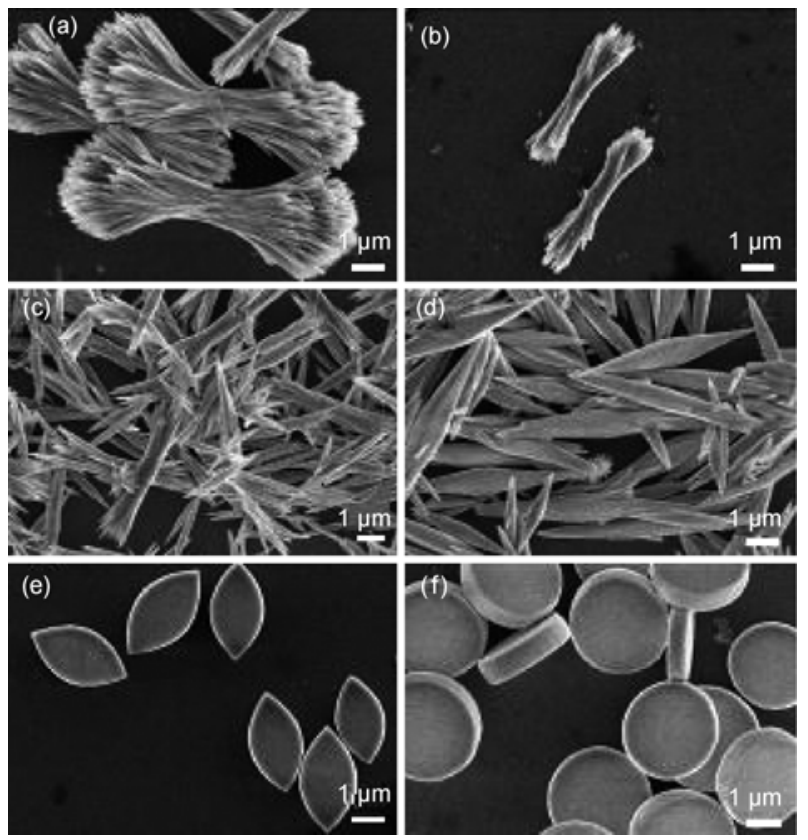

Figure 6 SEM images obtained from $\mathrm{Sb}_{8} \mathrm{O}_{11} \mathrm{Cl}_{2}\left(\mathrm{H}_{2} \mathrm{O}\right)_{6}$ samples with the different molar amounts of $\mathrm{Sb}^{3+}$ : (a) 0.90 , (b) 0.80 , (c) 0.70 , (d) 0.60 , (e) 0.53 and (f) $0.50 \mathrm{mmol}$. $0.25 \mathrm{~mol} / \mathrm{L} \mathrm{NaOH}$ was used. roles in regulating the shape evolution from sheaf-like to quasi-wafer structures. Figure 7 shows the schematic illustration of the growth process of $\mathrm{Sb}_{8} \mathrm{O}_{11} \mathrm{Cl}_{2}\left(\mathrm{H}_{2} \mathrm{O}\right)_{6}$ microcrystals with different morphologies. In the presence of PVP, when the ratio of $\mathrm{Sb}^{3+} / \mathrm{OH}^{-}$equals $1: 1.5$, crystal splitting occurs and the sheaf-like structure is formed. By changing the ratio to $1: 2.1$ the degree of crystal splitting decreases and the number of bundles in the sheaf-like morphology decreases. When the ratio of $\mathrm{Sb}^{3+} / \mathrm{OH}^{-}$reaches $1: 2.4$ and $1: 2.7$, simple crystal splitting is found and only a small quantity of fork-like features exist at the tips of the as-prepared products. The oval leaf-like and quasi-wafer structures showed no crystal splitting and this occurred when the ratio of $\mathrm{Sb}^{3+} / \mathrm{OH}^{-}$increased to $1: 2.88$ and $1: 3$, respectively.

Therefore, according to previous analysis, we postulate that both the addition of the surface stabilizer PVP and different concentrations of the reactants can affect the scale of crystal splitting, as illustrated for $\mathrm{Sb}_{8} \mathrm{O}_{11} \mathrm{Cl}_{2}\left(\mathrm{H}_{2} \mathrm{O}\right)_{6}$ microcrystals.

\section{Conclusions}

In this paper, we presented a mild and facile solution route for the shape-controlled synthesis of $\mathrm{Sb}_{8} \mathrm{O}_{11} \mathrm{Cl}_{2}\left(\mathrm{H}_{2} \mathrm{O}\right)_{6}$ microcrystals by simply changing the concentration of $\mathrm{NaOH}$ in the presence of PVP. A series of morphologies, such as sheaf-like, rhombic-plate, oval leaf-like and quasi-wafer were easily prepared by subtle manipulation of the concentration of $\mathrm{NaOH}$. With the proposed growth mechanism, we believe that this straightforward approach has the potential to guide shape-controlled synthesis of other inorganic crystals. In addition, antimony oxychlorides represent promising flame retardants because they include a halogen. Consequently, such compounds can be used as stand-alone flame retardants and provide good synergistic effects when used with a halogenated organic compound.

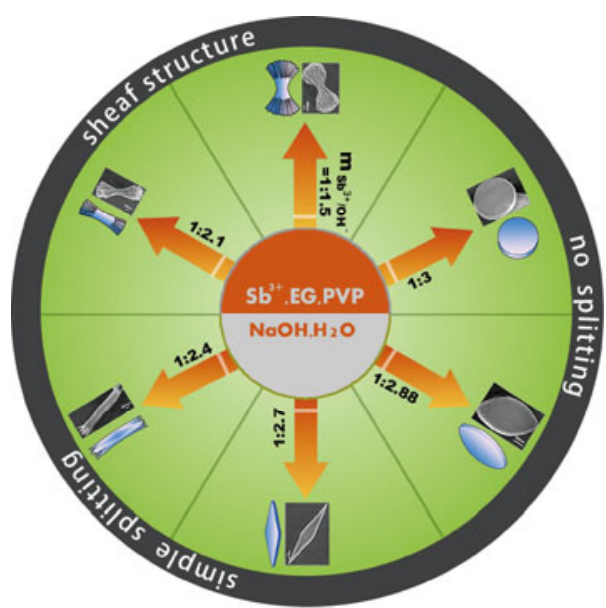

Figure 7 (Color online) Schematic illustration of the growth process of $\mathrm{Sb}_{8} \mathrm{O}_{11} \mathrm{Cl}_{2}\left(\mathrm{H}_{2} \mathrm{O}\right)_{6}$ microcrystals with different morphologies. 
This work was supported by the National Basic Research Program of China (2010CB93470) and the National Natural Science Foundation of China (50725208, 20973019).

1 Min Y, Akbulut M, Kristiansen K, et al. The role of interparticle and external forces in nanoparticle assembly. Nat Mater, 2008, 7: 527-538

2 Wang D, Lieber C M. Inorganic materials: Nanocrystals branch out. Nat Mater, 2003, 2: 355-356

3 Nguyen T D, Dinh C T, Do T O. Shape- and size-controlled synthesis of monoclinic ErOOH and cubic $\mathrm{Er}_{2} \mathrm{O}_{3}$ from micro- to nanostructures and their upconversion luminescence. ACS Nano, 2010, 4: 22632273

4 Jang S Y, Song Y M, Kim H S, et al. Three synthetic routes to singlecrystalline $\mathrm{PbS}$ nanowires with controlled growth direction and their electrical transport properties. ACS Nano, 2010, 4: 2391-2401

5 Gao J H, Gu H W, Xu B. Multifunctional magnetic nanoparticles: Design, synthesis, and biomedical applications. Acc Chem Res, 2009, 42: 1097-1107

6 Xiong Y J, Wiley B J, Xia Y N. Nanocrystals with unconventional shapes-A class of promising catalysts. Angew Chem Int Ed, 2007, 46: 7157-7159

7 Lu X B, Wen Z H, Li J H. Hydroxyl-containing antimony oxide bromide nanorods combined with chitosan for biosensors. Biomaterials, 2006, 27: 5740-5747

8 Li J H, Zhang J Z. Optical property and applications of hybrid semiconductor nanomaterials. Coordin Chem Rev, 2009, 253: 3015-3041

9 Costa L, Paganetto G, Bertelli G, et al. Thermal decomposition of antimony oxyhalides: I. Oxychlorides. J Therm Anal, 1990, 36: 11411153

10 Chen X Y, Huh H S, Lee S W. Hydrothermal synthesis of antimony oxychloride and oxide nanocrystals: $\mathrm{Sb}_{4} \mathrm{O}_{5} \mathrm{Cl}_{2}, \mathrm{Sb}_{8} \mathrm{O}_{11} \mathrm{Cl}_{2}$, and $\mathrm{Sb}_{2} \mathrm{O}_{3}$. J Solid State Chem, 2009, 181: 2127-2132

11 Tang J J, Wang Y, Jiao Z, et al. Self-assembly nanostructures of one-dimensional antimony oxide and oxychloride. Mater Lett, 2009, 63: 1481-1484

12 Li B J, Zhao Y B, Xu X M, et al. A simple method for the preparation of containing $\mathrm{Sb}$ nano- and microcrystallines via an ultrasound agitation. Ultraso Sonochem, 2007, 14: 557-562

13 Sui Y M, Fu W Y, Yang H B, et al. Low temperature synthesis of $\mathrm{Cu}_{2} \mathrm{O}$ crystals: Shape evolution and growth mechanism. Cryst Growth Des, 2010, 10: 99-108

14 Wang H H, Tian F, Li X P, et al. Preparation and shape evolution of cuprous oxide in the solution phases of copper (II) dodecyl sulfate. Powder Technol, 2010, 197: 298-302

15 Zhou J, Zhao H W, Li L D, et al. One-step synthesis and flame retardancy of sheaf-like microcrystal antimony oxychloride. J Nanosci Nanotech, 2011, doi: 10.1166/jnn.2011.4736

16 Tang J, Alivisatos A P. Crystal splitting in the growth of $\mathrm{Bi}_{2} \mathrm{~S}_{3}$. Nano Lett, 2006, 6: 2701-2706

17 Li L S, Sun N J, Huang Y Y, et al. Topotactic transformation of single-crystalline precursor discs into disc-Like $\mathrm{Bi}_{2} \mathrm{~S}_{3}$ nanorod networks. Adv Funct Mater, 2008, 18: 1194-1201

18 Tian L, Tan H Y, Vittal J J. Morphology-controlled synthesis of $\mathrm{Bi}_{2} \mathrm{~S}_{3}$ nanomaterials via singleand multiple-source approaches. Cryst Growth Des, 2008, 8: 734-738

$19 \mathrm{Hu} \mathrm{Y} \mathrm{H,} \mathrm{Chen} \mathrm{K} \mathrm{Z.} \mathrm{Crystal} \mathrm{splitting} \mathrm{in} \mathrm{the} \mathrm{growth} \mathrm{of} \beta-\mathrm{FeO}(\mathrm{OH})$. J Cryst Growth, 2007, 308: 185-188

20 Luo Z J, Li H M, Xia J X, et al. Controlled synthesis of different morphologies of $\mathrm{BaWO}_{4}$ crystals via a surfactant-assisted method. J Cryst Growth, 2007, 300: 523-529

21 He J H, Han M, Shen X P, et al. Crystal hierarchically splitting in growth of $\mathrm{BaWO}_{4}$ in positive cat-anionic microemulsion. J Cryst Growth, 2008, 310: 4581-4586

22 Deng $\mathrm{H}$, Liu C M, Yang S H, et al. Additive-mediated splitting of lanthanide orthovanadate nanocrystals in water: Morphological evolution from rods to sheaves and to spherulites. Cryst Growth Des, 2008, 8: 4432-4439

23 Chen G Y, Dneg B, Cai G B, et al. The fractal splitting growth of $\mathrm{Sb}_{2} \mathrm{~S}_{3}$ and $\mathrm{Sb}_{2} \mathrm{Se}_{3}$ hierarchical nanostructures. J Phys Chem C, 2008, 112: 672-679

24 Ota J, Roy P, Srivastava S K, et al. Morphology evolution of $\mathrm{Sb}_{2} \mathrm{~S}_{3}$ under hydrothermal conditions: Flowerlike structure to nanorods. Cryst Growth Des, 2008, 8: 2019-2023

25 Zhang M, Xu X D, Zhang M L. Hydrothermal synthesis of sheaf-like $\mathrm{CuO}$ via ionic liquids. Mater Lett, 2008, 62: 385-388

26 Pan Q T, Huang K, Ni S B, et al. Synthesis of sheaf-like CuO from aqueous solution and their application in lithium-ion batteries. $\mathrm{J}$ Alloys Comp, 2009, 484: 322-326

27 Kim S, Lee J S, Mitterbauer C, et al. Anomalous electrical conductivity of nanosheaves of $\mathrm{CeO}_{2}$. Chem Mater, 2009, 21: 1182-1186

28 Zhang F, Wong S S. Ambient large-scale template-mediated synthesis of high-aspect ratio single-crystalline, chemically doped rare-earth phosphate nanowires for bioimaging. ACS Nano, 2010, 4: 99-112

29 Liu K, You H P, Jia G, et al. Hierarchically nanostructured coordination polymer: Facile and rapid fabrication and tunable morphologies. Cryst Growth Des, 2010, 10: 790-797

Open Access This article is distributed under the terms of the Creative Commons Attribution License which permits any use, distribution, and reproduction in any medium, provided the original author(s) and source are credited. 